\title{
Violencia de género y otros factores asociados a la salud emocional de las usuarias del sector salud en México
}

\author{
Rosario Valdez-Santiago, M en Antrop, (I) Clara Juárez-Ramírez, M en Antrop, ${ }^{(I)} \vee$ Nelly Salgado-de Snyder, PhD, (I) \\ Carolina Agoff, Dra en Cs Soc, ${ }^{(2)}$ Leticia Avila-Burgos, Dra en Econ, (1) Martha C Hijar, PhD. ${ }^{(1)}$
}

\author{
Valdez-Santiago R, Juárez-Ramírez C, \\ Salgado-de Snyder VN, Agoff C, Avila-Burgos L, Híjar M. \\ Violencia de género y otros factores asociados \\ a la salud emocional de las usuarias \\ del sector salud en México. \\ Salud Publica Mex 2006;48 supl 2:S250-S258.
}

\begin{abstract}
Resumen
Objetivo. Identificar los factores asociados al malestar emocional en una muestra nacional de usuarias de servicios de salud del sector público: Secretaría de Salud (SSA), Instituto Mexicano del Seguro Social (IMSS), Instituto de Seguridad y Servicios Sociales de los Trabajadores del Estado (ISSSTE). Material y métodos. Se utilizó la base de datos de la Encuesta Nacional de Violencia contra las Mujeres en México, donde se aplicaron 26042 encuestas a usuarias de los servicios de salud de la SSA. Se utilizó la Escala de Salud Personal (ESP) para medir malestar emocional,; también se usó una escala de 19 reactivos que explora tipos de violencia y severidad de la misma. Mediante un modelo de regresión logística binaria se determinó la relación entre la presencia de malestar y la violencia de género, condiciones socioeconómicas y otras variables demográficas. Resultados. Entre los más relevantes está la identificación de la prevalencia de malestar emocional (I5.3\%) entre las mujeres que acuden a solicitar servicios de salud al sector público, y la relación de este malestar emocional generalizado con la experiencia de diferentes tipos de violencia de género. Los resultados sugieren que los factores asociados con el malestar emocional de la mujeres que recurren a los servicios de salud fueron: la edad (26 años o >); la actividad (jornalera o peona), y las horas laborales (7I a la semana o $>$ ); el consumo de alcohol (mayor consumo); el maltrato en la infancia (frecuencia y diversos tipos de maltrato); la seve-
\end{abstract}

Valdez-Santiago R, Juárez-Ramírez C, Salgado-de Snyder VN, Agoff C,Avila-Burgos L, Híjar M. Gender violence and other factors associated with emotional distress in female users of public health services in Mexico. Salud Publica Mex 2006;48 suppl 2:S250-S258.

\begin{abstract}
Objective. To identify and describe the factors associated with emotional distress in a national sample of women users of public health services in Mexico, such a Secretaria de Salud (SSA), Instituto Mexicano del Seguro Social (IMSS), Instituto de Seguridad y Servicios Sociales de los Trabajadores del Estado (ISSSTE). Material and Methods. This research study was conducted using the database of the National Survey of Violence against Women that consisted of the responses of a total of 26042 female users of health care services provided by the Mexican government health agencies. The Personal Health Scale (ESP per its initials in Spanish) was used to assess emotional distress. To measure violence a 19-item scale which explores different types of violence as well as severity was used. The relationship between emotional distress and gender violence was determined through a binary logistic regression model, as were economic status and demographic variables. Results. One of the most important findings of this study is the high prevalence of emotional distress (15.3\%) among women seeking health care services from the public sector and the relationship of such emotional distress with the experience of marital physical, psychological, and sexual violence. Factors associated with emotional distress among female users of health care services were age ( 26 and older); activity (laborer); working hours (7I hours a week or more); alcohol intake (greater intake); abuse during childhood (frequency
\end{abstract}

(I) Centro de Investigaciones en Sistemas de Salud, Instituto Nacional de Salud Pública. Cuernavaca, Morelos, México.

(2) Centro Regional de Investigaciones Multidisciplinarias. Universidad Nacional Autónoma de México. Cuernavaca, Morelos, México.

\footnotetext{
Fecha de recibido: 26 de agosto de 2004 - Fecha de aprobado: I 3 de diciembre de 2005 Solicitud de sobretiros: Dra. Leticia Avila Burgos, Instituto Nacional de Salud Pública, Avenida Universidad 655 Colonia Santa María Ahuacatitlán, 62508 Cuernavaca, Morelos, México Correo electrónico: lavila@correo.insp.mx
} 
ridad de la violencia de pareja (violencia severa), el estrato socioeconómico (muy bajo) y el tipo de localidad de residencia (localidades urbanas). Conclusiones. El predictor más importante del malestar emocional entre las usuarias del sector salud fue sufrir la violencia de pareja, sobre todo cuando ésta es severa, seguida de la violencia en la niñez. Así entonces, se propone utilizar herramientas de tamizaje en los servicios de salud, tanto de malestar emocional como de violencia intrafamiliar.Además, se propone diseñar y poner en marcha programas de atención y referencia de casos de malestar emocional femenino y de violencia intrafamiliar.

Palabras clave: violencia de pareja; salud emocional; servicios de salud; México and types of abuse); severity of marital violence (severe violence); socioeconomic status (very low SES); and type of dwelling (urban). Conclusions. The principal predictor of emotional distress was intimate partner abuse, especially in severe expression. The next predictor was violence in childhood. Taking into consideration these predictors it is recommended to use screening instruments to identify emotional distress and gender violence in health setting. It is important to design and implement attention and reference programs in public health services for women suffering from emotional distress and gender violence.

Keywords: intimate partner abuse; health services utilization; emotional distress; Mexico.
D iversos autores han señalado el impacto de la violencia de género en cualquiera de sus expresiones en la salud mental. Así, Koss, ${ }^{1}$ Díaz-Olavarrieta $^{2}$ y Nurius $^{3}$ señalan que las respuestas de la mayoría de las sobrevivientes a este tipo de violencia exhiben una reacción inmediata de malestar que, de no resolverse, las lleva a desarrollar patrones sintomáticos crónicos y de larga duración, consistentes en diferentes trastornos emocionales tales como el del estrés postraumático, la depresión, los problemas psicosomáticos y los trastornos de ansiedad, entre otros.

Lo anterior ha sido documentado en diferentes países donde los resultados de algunos estudios ${ }^{2,4-7}$ indican que las mujeres golpeadas tienen una probabilidad cuatro a cinco veces superior de necesitar tratamiento psiquiátrico que las no golpeadas, así como una probabilidad cinco veces mayor de intentar suicidarse.

Por otro lado, en situaciones de maltrato extremo las mujeres generan distorsiones cognitivas que les impiden reconocer la magnitud del peligro que corren tanto ellas como sus hijos, y les dificultan llevar a la práctica estrategias de protección que les permitan buscar ayuda. Esta situación es más trágica para aquellas mujeres que radican en lugares en donde no existen servicios comunitarios o institucionales que formen parte de su red de apoyo. ${ }^{8}$

Además, en el caso de las mujeres maltratadas se ha encontrado que vivir cotidianamente en una relación violenta es un predictor importante de alcoholismo. ${ }^{9}$ Incluso se ubica antes que el control de los ingresos, que la violencia en la familia de origen y que el estar unida con una pareja alcohólica. En estas mujeres, las drogas de mayor consumo son las prescritas por los médicos, lo cual es un indicador para el problema del malestar emocional y para la violencia de pareja. ${ }^{10}$ La medicalización de que son objeto las mujeres maltratadas que presentan sintomatología mental, es un problema ampliamente documentado en la literatura. ${ }^{11-14}$

Otro aspecto documentado es la relación entre enfermedades, como la depresión, y su relación con la violencia conyugal. Por ejemplo, en un estudio con 164 mujeres que tenían problemas severos con sus parejas y a quienes se les aplicó la Conflict Tactic Scale (CTS), se encontró que $28 \%$ de ellas transitaban en un rango de depresión de moderada a severa, mientras que $11 \%$ presentaron depresión severa. Un análisis multivariado de estos resultados relacionó la severidad de la depresión con las siguientes variables: abuso físico en la infancia, falta de apoyo cotidiano y abuso físico por parte de la pareja. ${ }^{15}$ Los hallazgos del estudio sugirieron a los autores que el abuso físico formaba parte importante de la etiología de la depresión en las mujeres maltratadas y que, combinado con la falta de apoyo en las actividades cotidianas, contribuía a disminuir la capacidad de respuesta de las mujeres ante el abuso, capacidad que, en otras circunstancias, surgiría como una habilidad "natural".

No obstante los estudios antes mencionados, la patología mental que pudieran padecer las mujeres maltratadas y que son atendidas en los servicios de salud raramente es identificada en el primer nivel de atención. Es por esto que las pruebas rápidas de tamizaje constituyen una herramienta importante para la identificación temprana de posibles casos. En este sentido, uno de los primeros síntomas de depresión es la manifestación de malestar emocional, que se caracteriza por la ausencia de afecto positivo y la presencia de síntomas aislados con severidad variable de cansancio excesivo, crisis de llanto, desesperanza y problemas somáticos. ${ }^{16}$

Debido a estos antecedentes, se consideró indispensable incluir, en el cuestionario de la primera Encuesta Nacional sobre Violencia contra las Mujeres en 
México 2003 (ENVIM 2003) ${ }^{17}$ una batería de preguntas, para explorar la relación entre el malestar emocional, las diferentes expresiones de la violencia hacia las mujeres y sus características sociodemográficas en el último mes previo a la entrevista. Esta información se exploró en una muestra nacional de usuarias de servicios de salud del sector público.

\section{Material y métodos}

En este trabajo se utilizaron las siguientes secciones del cuestionario de la ENVIM: características de la encuestada y de su hogar; salud general y personal de la encuestada; alcoholismo en la mujer; caracterización del maltrato.

Se aplicó la encuesta a 26042 mujeres. Para medir el malestar emocional se utilizó la Escala de Salud Personal (ESP) ${ }_{1}^{18}$ que desarrolló un grupo de profesionales de la salud mental en Nicaragua, como herramienta de tamizaje rápido de problemas de salud mental en clínicas del primer nivel de atención. Este instrumento identifica sintomatología asociada a una variedad de desórdenes mentales, incluyendo depresión, ansiedad y trauma. ${ }^{19}$

Originalmente, esta escala se diseñó tomando como base un cuestionario de autoreporte (Self Report Questionnaire -SRQ), diseñado y reportado por Penayo, Caldera y Jacobson, ${ }^{18}$ y Caldera, Kullgren, Penayo y Jacobson. ${ }^{19}$ Además, Ellsberg ${ }^{20}$ la utilizó en un estudio que realizó en Nicaragua para identificar el malestar emocional y su asociación con la violencia; por esta razón se consideró útil incluirla en la ENVIM.

Se sometió la escala a una serie de análisis, con el fin de determinar su utilidad y comportamiento para identificar posibles casos de malestar emocional en población mexicana. La ESP consta de 10 reactivos que hacen referencia a situaciones que pueden estar señalando la presencia de malestar emocional, por ejemplo: ¿Ha tenido dificultades para dormir? ¿Se ha sentido triste o afligida? ¿Se ha asustado o alarmado con facilidad? Estas preguntas se respondieron con cuatro opciones: $1=$ nunca, $2=$ pocas veces, $3=$ varias veces y $4=$ casi siempre.

La ESP se validó recientemente en México ${ }^{21}$ y en ese estudio se recomienda el punto de corte de 21.8 para identificar "posibles casos". Es decir, puntajes iguales o mayores al punto de corte indican la presencia de malestar emocional. En este estudio se aplicó la ESP a todas las mujeres encuestadas, antes de la exploración de las experiencias de violencia.

Se recurrió al análisis factorial, que permitió determinar el origen de la variación de los reactivos, así como su agrupamiento. Se identificaron tres factores que, en conjunto, explicaron $63.4 \%$ de la varianza de la ESP. Con la combinación lineal de estos factores se cons- truyó la variable dependiente, misma que se dicotomizó considerando la media grupal más una desviación estándar (16.3 $\pm 5.5=21.8)$. Así, a las mujeres que tuvieron puntajes por arriba de 22 se les consideró como casos, es decir, aquellas mujeres que presentaban síntomas cuya severidad indicaba la presencia de malestar emocional; las mujeres con calificación por debajo del punto de corte se consideraron como no casos.

Para la medición de la violencia de pareja se utilizó una escala de 19 reactivos que explora los diferentes tipos de violencia evaluados, a saber: la violencia psicológica, sexual y física. Se utilizó el análisis factorial para determinar el origen y agrupamiento de los reactivos; se obtuvieron cuatro factores que, en conjunto, explicaron $62.3 \%$ de la varianza. Para determinar la confiabilidad se calculó el alfa de Cronbach, que arrojó una consistencia de 0.99 . Con la combinación lineal de estos factores se construyó una variable, el Índice de Severidad de Violencia de Pareja (ISVP). Los puntos de corte utilizados fueron: $0=$ no violencia, la media más una desviación estándar como caso de violencia no severa, y la media más valores por arriba de una desviación estándar como casos de violencia severa. El ISVP entró al análisis como la principal variable independiente.

Los aspectos éticos de la encuesta se cubrieron informando a las mujeres seleccionadas los objetivos del estudio, y a quienes aceptaron participar, se les entregó una carta de consentimiento informado, garantizando la confidencialidad de la información. Asimismo, entrevistadoras capacitadas en el llenado del cuestionario y en el desarrollo adecuado de las entrevistas, se encargaron de llenar el cuestionario. Cada entrevista se llevó a cabo en un espacio privado donde sólo estaban la mujer y la entrevistadora. La tasa de respuesta fue de $98 \%$.

\section{Variables incluidas}

El malestar emocional fue la variable dependiente, dicotómica, categorizada como $0=$ "caso" de malestar emocional y $1=$ "no caso".

Las variables independientes fueron: edad, escolaridad, actividad laboral, ingreso, consumo de alcohol, estado civil, tipo de localidad (urbana o rural), y los antecedentes de maltrato durante la infancia, siendo ésta categorizada como: no tuvo maltrato, maltrato ocasional, varias veces, y casi siempre. Se construyó una variable indicadora de estrato socioeconómico a partir del análisis de componentes principales, incluyendo la escolaridad promedio de los integrantes del hogar, el número de aparatos electrodomésticos y el hacinamiento. La falta de información sobre el núme- 
ro de hijos de la entrevistada constituyó una limitante de importancia.

\section{Análisis estadístico}

En el análisis univariado se calcularon medidas de tendencia central y de dispersión para las variables continuas, así como porcentajes para las variables categóricas. Para el análisis bivariado se emplearon pruebas de ji cuadrada, t de Student, Mann-Whitney, y Kruskal-Wallis, según la escala de medición de las variables. Igualmente, se utilizó regresión logística simple, observando las razones de momios con sus intervalos de confianza y los valores $p$.

Finalmente, se realizó un modelo multivariado de regresión logística binaria. En su construcción se incluyeron aquellas variables que, en el análisis divariado, tuvieron un valor $p<0.25$; además, se incluyeron variables que conceptualmente fueran importantes para el análisis del problema de interés. Para las variables continuas como la edad y el salario se evaluó si el cambio en el logit de la variable dependiente se mantenía constante por cada incremento en la variable independiente (Boxtid Test).

Asimismo, se colapsaron categorías cada vez que este agrupamiento tuvo sentido teórico y se probó la no existencia de diferencias estadísticamente significativas entre ellas (Lincom Test). El ajuste del modelo se evaluó utilizando la prueba de bondad y ajuste del modelo; se consideró como ajuste adecuado cuando la significancia asociada a éste fue mayor de 0.10 . Se utilizó el paquete estadístico STATA, versión 8.2.*

\section{Resultados}

\section{Características de las mujeres}

Se entrevistaron 26042 usuarias de servicios de salud públicos. La prevalencia de malestar emocional en esta población fue de $15.6 \%$ ( $n=4.059)$.

Las que presentaron malestar emocional tienen significativamente mayor edad ( $39 \pm 14$ años) que las que no lo presentan $(35 \pm 13$ años) $(\mathrm{T} p<0.05)$. Asimismo, se observa que las que padecen de malestar tienen menor escolaridad, trabajan en mayor proporción fuera del hogar, consumen más frecuentemente alcohol, pertenecen a los estratos más bajos, notifican mayor violencia en la niñez y por parte de su pareja, en comparación con las que no lo presentan (cuadro I).

\footnotetext{
* StataCorp. STATA, Statistical Software 8.2.Texas: College Station, 2003.
}

\section{Relación ente malestar emocional y violencia de pareja}

De acuerdo con los resultados del análisis de datos del ISVP, $13.7 \%$ de las mujeres experimentan violencia no severa con su pareja actual y $7.04 \%$ violencia severa.

$\mathrm{Al}$ analizar la violencia de pareja y su asociación con el malestar emocional, se observó que todos los puntajes por tipos de violencia fueron significativamente más altos en las mujeres que presentaron malestar emocional. Esta asociación fue más evidente cuando se analizó la relación entre malestar emocional y grado de severidad de la violencia. Así por ejemplo, 23.3\% de las mujeres identificadas positivamente como "casos" de malestar emocional (CME) señalaron sufrir violencia psicológica severa en una proporción significativamente más alta que la encontrada en las que no presentaban malestar emocional $(8.3 \%)$.

Para el caso de la violencia sexual, $12.9 \%$ de las mujeres identificadas como CME señalaron sufrir de violencia sexual severa, proporción significativamente mayor $\left(\chi^{2} p=0.000\right)$ cuando se la compara con aquella de las que no tenían malestar emocional (3.7\%). Por otra parte, $14.5 \%$ de las mujeres identificadas como CME y $4.5 \%$ de las mujeres NCME señalaron sufrir violencia física; esta diferencia fue estadísticamente significativa $\left(\chi^{2} p=0.00\right)$ (figura 1$)$.

\section{Factores asociados al malestar emocional}

De acuerdo con los resultados, los factores asociados con el malestar emocional en las mujeres usuarias del sector salud fueron: la edad, la escolaridad, la actividad laboral, el total de horas trabajadas, la frecuencia de consumo de alcohol de la mujer, el antecedente de maltrato en la infancia, la severidad de la violencia con su pareja actual, el estrato socioeconómico y el tipo de localidad de residencia (cuadro II).

\section{Modelo multivariado}

Se formó con ocho efectos principales; si bien la escolaridad en la RMc es significativa, al incorporar la ocupación, aquélla, como los demás factores, pierde significancia. Cabe mencionar que no se incluyó a las mujeres mayores de 55 años, debido a que a esa edad es probable que existan otro tipo de problemas emocionales que pudieran estar confundiendo la asociación de la violencia de género con la salud mental.

Además, el efecto de la edad sobre los problemas emocionales no fue lineal (cuando se realizó la prueba de Box-Tidwell), por lo que si se incluía en escala continua se violaba ese supuesto de conformidad lineal. ${ }^{22}$ 


\section{Cuadro I \\ Características sociodemográficas \\ DE LAS USUARIAS DE SERVICIOS DE SALUD PÚBLICOS. \\ Encuesta Nacional sobre Violencia contra las Mujeres 2003. México}

\begin{tabular}{|c|c|c|c|c|c|}
\hline & \multicolumn{2}{|c|}{$\begin{array}{l}\text { Malestar } \\
N=4059\end{array}$} & \multicolumn{2}{|c|}{$\begin{array}{c}\text { Sin malestar } \\
N=21983\end{array}$} & \multirow[t]{2}{*}{$P$} \\
\hline & $N$ & $\%$ & $\mathrm{~N}$ & $\%$ & \\
\hline \multicolumn{6}{|l|}{ Edad (años cumplidos) } \\
\hline 15 a 25 & 658 & 19 & 6332 & 31.7 & 0.00 \\
\hline 26 a 55 & 2792 & 80.9 & 13640 & 68.3 & \\
\hline \multicolumn{6}{|l|}{ Escolaridad } \\
\hline Analfabeta & 551 & 13.6 & I 884 & 8.6 & \\
\hline Primaria & 1782 & 43.9 & 8468 & 38.5 & 0.00 \\
\hline Secundaria & 881 & 21.7 & 6115 & 27.8 & \\
\hline Preparatoria y más & 845 & 20.8 & 5516 & 25.1 & \\
\hline \multicolumn{6}{|l|}{ Actividad que realiza } \\
\hline Estudiante & 44 & 1.1 & 522 & 2.4 & \\
\hline Trabajadora sin paga & 60 & 1.5 & 288 & 1.3 & \\
\hline Ama de casa & 1642 & 40.6 & 10030 & 45.7 & 0.00 \\
\hline Desempleada & 694 & 17.2 & 3757 & 17.1 & \\
\hline Jornalera o peona & 43 & 1.1 & 148 & 0.7 & \\
\hline Cuenta propia & 469 & 11.6 & 1750 & 8 & \\
\hline Empleada & 1090 & 27 & 5430 & 24.8 & \\
\hline
\end{tabular}

Horas trabajadas (trabajo doméstico y en el sector productivo)

\begin{tabular}{lrrrrr}
50 y menos & 2457 & 60.5 & 14313 & 65.1 & 0.00 \\
\hline 51 a 70 & 958 & 23.6 & 4959 & 22.6 & \\
\hline 71 y más & 644 & 15.9 & 2711 & 12.3
\end{tabular}

Frecuencia del consumo de alcohol

\begin{tabular}{lrrrrr}
$>$ I vez al mes & 238 & 5.9 & 565 & 2.6 & \\
\hline Ocasionalmente & I 966 & 49 & 9394 & 43.2 & 0.00 \\
\hline No consume & I 808 & 45. I & || 763 & 54.2 &
\end{tabular}

Antecedentes de maltrato en la niñez

\begin{tabular}{|c|c|c|c|c|c|}
\hline No le pegaban & 1743 & 43.1 & 13001 & 59.3 & \\
\hline Ocasionalmente & 1014 & 25.1 & 5460 & 24.9 & 0.00 \\
\hline Varias veces a casi siempre & 1285 & 31.8 & 3453 & 15.8 & \\
\hline \multicolumn{6}{|c|}{ Índice de severidad de violencia por pareja actual } \\
\hline Sin violencia & 2358 & 62 & 17412 & 82.3 & \\
\hline Violencia no severa & 790 & 20.8 & 2640 & 12.5 & 0.00 \\
\hline Violencia severa & 656 & 17.2 & 1102 & 5.2 & \\
\hline
\end{tabular}

Estrato socioeconómico

\begin{tabular}{lrrrrr} 
Muy bajo & I 917 & 47.3 & 9836 & 44.9 & \\
\hline Bajo & I 550 & 38.3 & 8630 & 39.4 & 0.00 \\
\hline Medio & 463 & $\mid 1.4$ & 2557 & $\mid 1.7$ & \\
\hline Alto & 120 & 3 & $89 \mid$ & 4.1
\end{tabular}

Tipo de localidad

\begin{tabular}{lrrrrr} 
Rural & 366 & 9 & 2688 & 12.2 & 0.00 \\
\hline Urbana & 3693 & 91 & 19295 & 87.8
\end{tabular}

Fuente: Cuestionario de la ENVIM, 2003

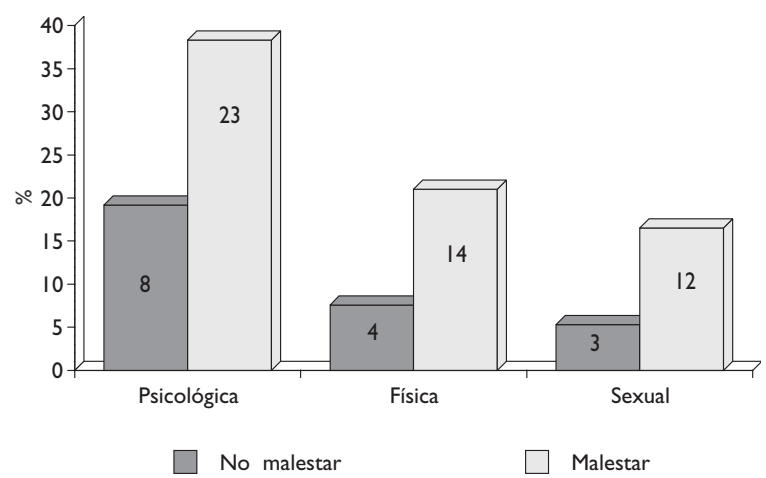

Figura I.Violencia de pareja, SEgún tipo y Malestar EMOCIONAL DE LAS USUARIAS DE SERVICIOS DE SALUD. Encuesta Nacional sobre Violencia contra las MuJeRes 2003. México

Asimismo, se probaron las interacciones, sin encontrar significancia estadística en ninguna de ellas.

Los resultados del modelo se presentan en el cuadro III, donde se observa que la violencia de pareja actual fue el predictor más importante de malestar emocional, ya que cuando ésta se notifica como no severa, la posibilidad de sufrir malestar emocional casi se duplica cuando se la compara con la de las mujeres que no señalan violencia (RM 1.99, IC95\% 1.81 a 2.2); pero si esta violencia es severa, la posibilidad de tener malestar emocional se incrementa 3.55 veces (RM 3.55, IC95\% 3.17 a 3.99) con relación a la de las mujeres que no tienen una relación de pareja violenta.

Por otro lado, las mujeres con antecedentes de maltrato físico o emocional en la niñez presentaron una mayor posibilidad de sufrir malestar emocional en la vida adulta; así, las mujeres que refirieron haber sido golpeadas, insultadas o humilladas de manera ocasional durante la infancia, tuvieron una posibilidad $25 \%$ superior en relación con las que refirieron no haber sido golpeadas. Sin embargo, si el maltrato infantil se presentó de "varias veces" a "casi siempre", dicha posibilidad se incrementó a más del doble (RM 2.21, IC95\% 2.01 a 2.42) en relación con la categoría de referencia (cuadro III).

Respecto a las variables sociodemográficas asociadas al malestar emocional, se observa que las mujeres de 26 años o más tienen una posibilidad (RM 1.89, IC95\% 1.71-2.01) mayor (89\%) de sufrir malestar emocional en relación con el grupo más joven (15 a 25 años) cuadro III.

La actividad laboral se notificó significativa sólo para tres categorías: las amas de casa, las jornaleras 
Cuadro II

FACTORES ASOCIADOS CON VIOLENCIA DE PAREJA Y MALESTAR EMOCIONAL EN USUARIAS DE SERVICIOS de salud. Encuesta Nacional de Violencia contra las Mujeres 2003. México

RM Cruda IC $95 \%$

Edad (años cumplidos)

\begin{tabular}{llc}
15 a 25 & 1 & - \\
\hline 26 a 55 & 1.97 & $1.8-2.15$
\end{tabular}

Escolaridad

Preparatoria y más

\begin{tabular}{lcc} 
Preparatoria $y$ más & \multicolumn{1}{c}{-} \\
\hline Secundaria & 0.94 & $0.85-1.04$ \\
\hline Analfabeta & 1.34 & $1.26-1.50$ \\
\hline
\end{tabular}

Actividad que realiza

\begin{tabular}{llc} 
Empleada & $\mathrm{I}$ & - \\
\hline Cuenta propia & $\mathrm{I} .33$ & $\mathrm{I} .18-\mathrm{I} .5$ \\
\hline Jornalera o peona & $\mathrm{I} .45$ & $\mathrm{I} .02-2.04$ \\
\hline Desempleada & 0.92 & $0.83-1.02$ \\
\hline Ama de casa & 0.82 & $0.75-0.89$ \\
\hline Trabajadora sin paga & 1.04 & $0.78-1.4$ \\
\hline Estudiante & 0.42 & $0.31-57$
\end{tabular}

Horas trabajadas (trabajo doméstico y en el sector productivo)

\begin{tabular}{llc}
50 y menos & I & - \\
\hline 51 a 70 & 1.38 & $1.26-1.52$ \\
\hline 71 y más & 1.25 & $1.04-1.22$
\end{tabular}

Frecuencia del consumo de alcohol

\begin{tabular}{lll} 
No consume & I & - \\
\hline Ocasionalmente & 1.36 & $1.27-1.46$ \\
\hline$>$ I vez al mes & 2.74 & $2.33-3.2$
\end{tabular}

Antecedentes de maltrato en la niñez

\begin{tabular}{llc} 
No le pegaban & I & - \\
\hline Ocasionalmente & I.38 & I.27-I.5I \\
\hline Varias veces a casi siempre & 2.78 & $2.56-3.0 \mathrm{I}$
\end{tabular}

Índice de severidad de violencia por pareja actual

\begin{tabular}{llc} 
Sin violencia & $\mathrm{I}$ & - \\
\hline Violencia no severa & 2.21 & $2.02-2.42$ \\
\hline Violencia severa & 4.39 & $3.95-4.89$
\end{tabular}

Estrato socioeconómico

\begin{tabular}{llc} 
Alto & $\mathrm{I}$ & - \\
\hline Bajo & 1.34 & $\mathrm{I} .08-\mathrm{I} .67$ \\
\hline Medio & 1.33 & $\mathrm{I} .09-\mathrm{I} .63$ \\
\hline Muy bajo & $\mathrm{I} .47$ & $\mathrm{I} .19-\mathrm{I} .76$
\end{tabular}

Tipo de localidad

\begin{tabular}{llc} 
Rural & I & - \\
\hline Urbana & I.4I & $1.25-1.58$
\end{tabular}

Fuente: Cuestionario de la ENVIM, 2003
Cuadro III

Modelo de Regresión logística multivariado.*

Factores ASOCIADOS CON VIOLENCIA DE PAREJA

Y MALESTAR EMOCIONAL EN USUARIAS DE SERVICIOS

de salud. Encuesta Nacional de Violencia contra las Mujeres 2003. México

Variables

RM ajustada IC $95 \% \quad P$

Edad (años cumplidos)

\begin{tabular}{llll}
15 a 25 años & I* & & \\
\hline 26 a 55 años & 1.89 & $|.7|-2.01$ & 0.00
\end{tabular}

Actividad laboral

\begin{tabular}{llll} 
Empleada & $\mathrm{I}^{*}$ & & \\
\hline Cuenta propia & $\mathrm{I} .17$ & $1.02-1.34$ & 0.02 \\
\hline Jornalera o peona & 1.46 & $0.99-2.16$ & 0.06 \\
\hline Desempleada & 0.96 & $0.84-1.09$ & 0.53 \\
\hline Ama de casa & 0.89 & $0.80-0.99$ & 0.03 \\
\hline Trabajadora sin paga & 1.10 & $0.80-1.50$ & 0.53 \\
\hline Estudiante & 0.92 & $0.64-1.33$ & 0.68
\end{tabular}

Frecuencia de consumo de alcohol de la mujer

\begin{tabular}{llll} 
No consume alcohol & I* $^{*}$ & & \\
\hline Ocasionalmente & $\mathrm{I} .25$ & $\mathrm{I} .16-1.35$ & 0.00 \\
\hline Más de I vez al mes & 2.19 & $\mathrm{I} .84-2.62$ & 0.00
\end{tabular}

Horas totales trabajadas (trabajo doméstico y en el sector productivo)

\begin{tabular}{llll}
50 y menos & I* & & \\
\hline 51 a 70 & 1.05 & $0.95-1.15$ & 0.35 \\
\hline 71 y más & 1.26 & $1.13-1.42$ & 0.00
\end{tabular}

Antecedentes de maltrato en la niñez

\begin{tabular}{llll} 
No le pegaban & I* $^{*}$ & & \\
\hline Ocasionalmente & I.25 & I.I4-I.37 & 0.00 \\
\hline Varias veces a casi siempre & $2.2 \mathrm{I}$ & $2.0 \mathrm{I}-2.42$ & 0.00
\end{tabular}

Índice de severidad de violencia de pareja actual

\begin{tabular}{llll} 
Sin violencia & I* & & \\
\hline Violencia & 1.99 & $1.8 \mathrm{I}-2.20$ & 0.00 \\
\hline Violencia severa & 3.55 & $3.17-3.99$ & 0.00
\end{tabular}

Estrato socioeconómico

\begin{tabular}{llll} 
Alto & $\mathrm{I}^{*}$ & & \\
\hline Medio & $\mathrm{I} .34$ & $\mathrm{I} .06-\mathrm{I} .69$ & $0.0 \mathrm{I}$ \\
\hline Bajo & $\mathrm{I} .29$ & $\mathrm{I} .04-\mathrm{I} .6 \mathrm{I}$ & 0.02 \\
\hline Muy bajo & $\mathrm{I} .43$ & $\mathrm{I} . \mathrm{I} 5-\mathrm{I} .77$ & 0.00
\end{tabular}

Tipo de localidad

\begin{tabular}{llll} 
Rural & I* & & \\
\hline Urbana & I.58 & I.38-I.8 & 0.00
\end{tabular}

*Bondad de ajuste $p=0.103$

Fuente: Cuestionario de la ENVIM, 2003 
o peonas y las que trabajaban por cuenta propia, aunque el sentido de la asociación es contrastante dependiendo de la categoría. Así, el ser ama de casa es un factor que protege contra el malestar emocional (RM 0.89, IC95\% .8-.99) cuando se compara con el ser empleada, mientras que el ser trabajadora por cuenta propia o jornalera se asoció con una posibilidad 17 y 46\% mayor, respectivamente, de presentar malestar emocional, en comparación con las empleadas (cuadro I).

El total de horas que las mujeres trabajaban, incluyendo no sólo la jornada de trabajo fuera del domicilio, sino también el trabajo doméstico no remunerado, fue un factor de riesgo para presentar malestar emocional. Así, las mujeres que trabajaban más de 71 horas a la semana tuvieron una posibilidad 26\% (RM 1.26, IC95\% 1.13 a 1.37) mayor de presentar malestar emocional, en comparación con las que trabajaban 50 horas a la semana y menos. Para las mujeres que trabajaban entre $50 \mathrm{y}$ 70 horas no se encontró diferencia significativa.

El estrato socioeconómico fue un factor que también se asoció significativamente al malestar emocional. Así, las mujeres que pertenecían al estrato muy bajo tuvieron una posibilidad 43\% (RM 1.43, IC95\% 1.15 a 1.77) mayor de ser "caso" que las que pertenecían al estrato alto. Para las que pertenecían al estrato bajo esta posibilidad fue de 29\% (RM 1.29, IC95\% 1.04 a 1.61), y para las que pertenecían al medio fue de 34\% (RM 1.34, IC95\% 1.06 a 1.69), en comparación con las que se ubicaron en el estrato alto.

En relación con el tipo de localidad de residencia de las entrevistadas, las mujeres que vivían en comunidades urbanas tuvieron una posibilidad $58 \%$ más alta de tener malestar emocional, en comparación con las que vivían en comunidades rurales (RM 1.58, IC95\% 1.38 a 1.8).

Por último, la mayor frecuencia de consumo de alcohol por parte de las mujeres se encontró asociada con una mayor posibilidad de tener malestar emocional. Así, para las que consumían alcohol ocasionalmente la posibilidad de tener problemas emocionales fue $25 \%$ mayor en relación con las que no lo consumían, pero si este consumo se incrementaba a una vez o más al mes, la posibilidad era de más del doble (cuadro III). Las variables como educación y salario mínimo no se indicaron como significativas.

\section{Discusión}

La identificación de una prevalencia nacional de 15.6\% de malestar emocional entre las mujeres que acuden al sector salud público es uno de los primeros hallazgos de la ENVIM en el área de salud mental. Este dato merece una reflexión especial, ya que indica la existencia de un malestar femenino generalizado más allá de una enfermedad emocional específica (como la depresión y los estados de ansiedad). En este sentido, vale la pena articular estos hallazgos con la definición de malestar emocional de las mujeres, que autoras como Burín y col. conceptualizan como "el sufrimiento femenino, ligado a una situación específica de subordinación que se manifiesta a través de trastornos mentales". ${ }^{11}$ Este aspecto cobra mayor significado al revisar las variables asociadas a dicho malestar y que lo conforman, sobre todo las relacionadas con las diferentes expresiones de violencia y su severidad ejercidas en contra de las mujeres en la relación de pareja.

En este tenor, los resultados de la ENVIM ponen en evidencia la estrecha asociación que existe entre el malestar emocional generalizado y la violencia de pareja, y que se comprueba al ser ésta el predictor más importante para el malestar emocional. Lo anterior concuerda con los hallazgos en la literatura nacional ${ }^{23}$ e internacional, ${ }^{24-26}$ donde se notifica haber encontrado que las principales consecuencias emocionales de sufrir cualquier tipo de maltrato son una autopercepción de vulnerabilidad e indefensión, que se manifiesta en síntomas depresivos como una expresión del malestar cotidiano al que están expuestas las mujeres que sufren de maltrato por parte de la pareja. ${ }^{27}$

Al respecto, los resultados de la ENVIM proporcionan elementos para seguir documentando que el origen de los malestares cotidianos en las mujeres radica en la construcción social de la identidad genérica y en la inequidad intergenérica. En este sentido, el malestar adquiere significado en la medida en que produce una modificación en la vida cotidiana de estas mujeres y altera su noción sobre sí mismas. ${ }^{28}$

Resulta especialmente relevante el resultado de la ENVIM en relación con la violencia en la infancia como un importante predictor de malestar emocional en la vida adulta, pues se ha señalado la utilización de la violencia en la familia como mecanismo para inculcar a los hijos y a las mujeres los principios de la jerarquía genérica y generacional. ${ }^{29}$ Esta experiencia temprana de violencia se va acumulando a lo largo del ciclo de vida y tiene una fuerte asociación en el malestar emocional de las mujeres en la vida adulta. ${ }^{30}$

Sobre este aspecto diversos autores ${ }^{3,14,27}$ señalan la naturaleza de los síntomas y los patrones de padecimientos mentales asociados a las diferentes experiencias de violencia; por ejemplo, las mujeres que tuvieron experiencias de maltrato infantil, presentan en la vida adulta más padecimientos de orden mental. La combinación de experiencias de maltrato infantil con maltrato en la vida adulta también tuvo una mayor correlación con enfermedades y padecimientos de orden mental que 
aquellas que sólo tuvieron maltrato infantil o sólo maltrato en la edad adulta. ${ }^{4-6}$

De otra parte, el hecho de que las mujeres mayores tengan más posibilidad de presentar malestar emocional, coincide con lo notificado en la literatura especializada sobre los efectos acumulativos de la violencia ${ }^{31}$ que señala que aquellas mujeres que han tenido una relación larga de maltrato presentan aspectos negativos sobre su persona que afectan a diferentes dimensiones de su salud, su capacidad productiva y su inserción en la vida social.

Además, se ha documentado que una de las razones que esgrimen las mujeres de edad adulta (mayores de 55 años) para seguir conviviendo con una pareja que las maltrata, es que a esa edad tienen diversos padecimientos que las hacen percibirse físicamente limitadas para buscar otras opciones. Estas mujeres manifestaron que cuando el maltrato se inició en su relación de pareja, ellas se acercaron a pedir ayuda a diversas instituciones sociales, pero recibieron una respuesta no satisfactoria. Asimismo, expresaron que vivir con una pareja abusiva no les permitió desarrollar las habilidades necesarias para conseguir un trabajo remunerado. ${ }^{31}$

Respecto a las condiciones sociodemográficas relacionadas con el malestar emocional, mención especial amerita el hecho de que las mujeres que señalaron trabajar 71 horas y más a la semana, tanto en actividad laboral como en el trabajo doméstico, tuvieron mayor riesgo de sufrir malestar emocional. Lo anterior pone en evidencia las condiciones laborales de desventaja en las que se desempeñan las mujeres, tales como los horarios prolongados sin periodos de descanso, carencia de beneficios de ley, explotación económica y segregación salarial, además de la doble o triple jornada, situación que mina sus condiciones físicas y mentales. ${ }^{32,33}$

Las jornaleras y peonas, que, además de estar obligadas a enfrentar las condiciones laborales antes descritas, deben realizar cotidianamente los trabajos domésticos o incluso el trabajo de la parcela familiar, tienen una posibilidad $46 \%$ superior -según resultados de la ENVIM- de sufrir malestar emocional, en comparación con las empleadas en trabajos no relacionados con la agricultura. ${ }^{17}$

$\mathrm{Al}$ respecto, existe información sobre las condiciones sociales de vulnerabilidad en las que se desempeñan las trabajadoras agrícolas, aspectos que ayudan a comprender los resultados de la ENVIM al señalar que estas mujeres están expuestas a jornadas laborales de más de 12 horas en condiciones extenuantes (como trabajar de pie o agachadas), no tienen horarios determinados y sufren la variabilidad de las condiciones climáticas, además de vivir en condiciones deplorables para su salud y la de sus familias y de percibir ingresos menores que los jornaleros que trabajan en sus mismas condiciones. Lo anterior, aunado a la experiencia de maltrato por parte de la pareja, señala la necesidad de crear programas de apoyo que atiendan estos aspectos. ${ }^{33}$

Por último, es importante señalar algunas limitaciones del estudio. La primera se refiere a la generalización de los resultados, ya que debido a que en la ENVIM sólo se incluyen usuarias de servicios de salud públicos estos hallazgos no son extrapolables a la población general. Además, se puede suponer una mayor prevalencia de malestar emocional entre las usuarias del sector salud público debido a la asociación que se ha señalado entre una mayor auto-percepción de problemas de salud y malestar emocional. ${ }^{21}$

Otra consideración importante es lo referente a la definición de "caso" y "no caso" de malestar emocional. Si bien sería necesaria una confirmación clínica, este tipo de estudio demuestra su utilidad al usar una escala que permite identificar a un grupo de mujeres que presentan mayor riesgo y que podrían ser focalizadas para un mejor diagnóstico y manejo. A pesar de ello, las asociaciones encontradas entre malestar emocional, violencia de género y los otros factores asociados, siguen siendo válidas.

Por último, debido a que se trata de un estudio transversal, existe el problema de la ambigüedad temporal $^{34}$ para todas las asociaciones identificadas, por lo que sería recomendable realizar otros estudios de tipo longitudinal.

\section{Conclusiones}

A partir de los hallazgos del estudio resulta evidente la necesidad de llevar a cabo una identificación rutinaria de mujeres que manifiestan malestar emocional en la consulta del primer nivel de atención, para lo cual se puede utilizar la Escala de Salud Personal, validada en el país, y que constituye uno de los aportes metodológicos de la ENVIM 2003. ${ }^{21}$ Esta identificación llevaría a desarrollar programas para la atención, en los servicios de salud, del malestar emocional femenino, que incluirían estrategias de capacitación del personal de salud y la implantación de módulos de atención especializada en salud mental, además de promover acciones comunitarias de promoción de la salud mental, por señalar algunas.

Asimismo, es necesario garantizar la identificación, atención y referencia de las mujeres que sufren violencia de pareja actualmente, así como de aquellas que hayan sufrido algún tipo de violencia en la infancia; es éste uno de los objetivos de la Norma Oficial Mexicana NOM-190-SSA1-1999, "Prestación de servicios de salud. Criterios para la atención Médica de la Violencia Familiar". ${ }^{35}$ 


\section{Referencias}

I. Koss MP.The women's mental health research agenda:Violence against women. Am Psychol 1990; 45:374-380.

2. Díaz-Olavarrieta C, Ellertson C, Paz F, De León S, Alarcón-Segovia D. Prevalence of battering among I 780 outpatients at an internal medicine institution in Mexico. Soc Sci Med 2002;55:1589-1602.

3. Nurius P, Macy R, Bhuyan R, Holt V. Contextualizing depression and physical functioning in battered women.J Interpers Violence 2003; 18 (I2): | 14||$-|43|$.

4. Jaffe P,Wolfe D, Wilson SW, Zak L. Emotional and physical health problems of battered women. Am J Psychiatry 1986;121:74-77.

5. Kubany E, McKenzie W, Owens J, Leisen M, Kaplan A, Pavich E. PTSD among women survivors of domestic violence in Hawaii. Hawaii Med J. 1996; 55 (9): 164-165.

6. Campbell JC, Kub JE, Rose L. Depression in battered women. JAMA 1996; $51(3): 100-106$

7. Counts DA. Female suicide and wife abuse: $A$ cross-cultural perspective. Suicide Life Threat Behav 1987; 17 (3): 194-204. 8. Freyermuth G. Las mujeres de humo. Morir en Chenalhó. Género, etnia y generación, factores constitutivos del riesgo durante la maternidad. México: CIESAS/ Instituto de la Mujer/ Instituto Nacional de las Mujeres/ ACASAC/Miguel Ángel Porrúa, 2003.

9. Casco F, Natera G. El alcoholismo en la mujer: la explicación que ellas mismas dan. Salud Mental 1993;16(I): 22-27.

10. Ramos L, Saltijeral MT, Caballero MA. Impacto de la violencia en la salud mental. Estado actual y perspectivas. Salud Mental 1996;

Suplemento Abril: 19-29, 1996.

II. Burín M, Moncarz E, Velásquez S. El malestar de las mujeres. La tranquilidad recetada. Buenos Aires: Paidós, 1990.

12. Gleason WJ. Mental disorders in battered women:An empirical study.Violence Vict 1993;8:50-68.

13. Orava TA, McLeod PJ, Sharpe D. Perceptions of control, depressive symptomatology, and self-esteem of women in transition from abusive relationships. J Fam Violence 1996; I (2):65-77.

14. Roberts GL, Gail WL, Lawrence JM. How does domestic affect women's mental health? Women Health 1998;28(I): I63-I72.

15. Campbell J, Harris MI, Lee RK.Violence research:An overview. Scholarly inquiry for nursing practice. Int J Nurs Stud 1995;9(2): 105-I I6. 16. Salgado de Snyder VN, Maldonado M. Características psicométricas de la escala de depresión del Centro de Estudios Epidemiológicos en mujeres mexicanas adultas de áreas rurales. Salud Publica Mex 1994; 36(2):200-209.

17. Olaiz G, Rico B, Del Río A. Encuesta Nacional Sobre Violencia contra las Mujeres 2003. Cuernavaca Mor., México: Instituto Nacional de Salud Pública 2004

18. Penayo U, Caldera T, Jacobson L. Prevalencia de trastornos mentales en adultos de Subtiava, León, Nicaragua. Boletita de la Oficina Panamericana de Salud II3:I37-I49.

19. Caldera JT, Kullgren G, Penayo U, Jacobson I. Is treatment in groups a useful alternative for psychiatry in low-income countries? An evaluation of a psychiatric outpatient unit in Nicaragua. Acta Psychiatr Scand 1995; 92: 386-391.

20. Ellsberg M, Caldera T, Herrera A,Winkvist A, Kullgren G. Domestic violence and emotional distress among Nicaraguan women: Results from a population-based study. Am Psy 1999;54:30-36.

2I.Valdez-Santiago R, Salgado de Snyder N. ESP, una escala breve para identificar malestar emocional en la práctica médica de primer nivel: características psicométricas. Salud Mental 2004; 27: 55-62.

22. Bagley S, White H, Golomb B. Logistic regression in the medical literature: standards for use and reporting, with particular attention to one medical domain. J Clin Epidemiol 2001;54:979-985.

23.Valdez-Santiago R, Shrader E. Características y análisis de la violencia doméstica en México: el caso de una micro-región de ciudad

Nezahualcóyotl. En: Aún la luna a veces tiene miedo. México: Centro de Investigación y Lucha contra la Violencia Doméstica, A.C. (CECOVID A.C.), 1992:33-49.

24. Heise L, Pitanguy J, Germain A. Violencia contra la mujer: La carga oculta sobre la salud. Programa Mujer, Salud y Desarrollo. Washington, D.C.: Organización Panamericana de la Salud, 1994.

25. Stark E, Flitcraft A. Spouse abuse. En:Violence in America:A Public Health Aproach. Nueva York: Oxford University Press, 1991.

26. Jaffe P,Wolfe D, Wilson S, Zak I. Emotional and physical health problems of battered women. Am J Psychiatry 1986; 121:74-77.

27. Saunders D. Posttraumatic stress symptom, profiles of battered women: A comparison of survivors in two settings. Violence Vict 1994;9 (I): 29-43.

28. Dio-Bleichmar E. La depresión en la mujer. Barcelona: Ediciones Temas de Hoy, 1991.

29. Freyermuth G. La violencia de género como factor de riesgo en la maternidad. En: Martha Torres comp. Violencia contra las mujeres en contextos urbanos y rurales. México D. F. :El Colegio de México, 2004. 30. Tammy O. Perceptions of control, depressive symptomatology, and self-esteem of women in transition from abusive relationships.J Fam Violence 1996; II(2):42-5I.

3I. Zink T, Regan S, Jacobson C, Pabst S. Hidden Victims: The Healthcare Needs and Experiences of Older Woomen in Abusive Relationships. A qualitative study of older women's reasons for remaining in abusive relationships. Violence Against Women \& Health 2004;8(I3):898-908. 32. Rangel G. Problemática actual de las trabajadoras asalariadas: vulnerabilidad de los niveles de bienestar (serie de Cuadernos de Trabajo No.5). México: GIMTRAP/UNIFEM, 1998.

33. Acevedo M, Lara MA. Salud mental y trabajo extradoméstico. En: Lara MA, Salgado de Snyder VN, comp. La salud mental de las mujeres mexicanas (Cálmese son sus nervios, tómese un tesito). México: Ed. Pax, 2002: I3I-I50.

34. Hosmer D, Lemeshow S. Applied logistic regression. 2a.edición. Nueva York: David W. Hosmer, Stanley Lemeshow, 2000.

35. Secretaría de Salud. Norma Oficial Mexicana NOM-190-SSA I1999. Prestación de servicios de salud. Criterios para la atención médica de la violencia intrafamiliar. Diario Oficial de la Federación. México D.F. Marzo 2000. 